\title{
Function-Oriented Dimensional Metrology - More than Determining Size and Shape
}

\author{
Wito Hartmann ${ }^{1}$, Albert Weckenmann ${ }^{1}$ \\ ${ }^{1}$ Chair Quality Management and Manufacturing Metrology, University Erlangen-Nuremburg, Germany
}

\begin{abstract}
:
Today's dimensional metrology has undergone some generational changes. Looking back in history, manufacturing functional products without metrology was just a trial and error procedure, so only a small number of simple products were produced. First, with the implementation of simple gage blocks the construction of complex buildings such as the pyramids were possible. Later, during the industrial revolution requirements for mass production increased, so simple 2-point measurements were no longer sufficient to ensure mating ability and interchangeability of more and more intricate components. Systems for geometrical dimensioning and tolerancing (GD\&T) have been introduced, which enable the geometrical description of complex product properties, and also measurement instruments were developed further from simple manual measurement instruments to high-resolution multi-sensor coordinate measuring machines, which combine tactile, optical and x-ray-tomographic measurement principles. Currently, another paradigm shift is imminent: Conformance proving is changing from purely geometry oriented measurements to function oriented virtually testing. By modeling and simulating the function of a component it is possible to evaluate the functional ability of a component based on dimensional measurement data. This leads to an unambiguous product specification, a better process understanding, a verification result based directly on function-oriented parameters instead of indirectly function related dimensional parameters and so to a more veridic prediction of functional ability.
\end{abstract}

Key words: dimensional metrology, function-oriented parameters, GD\&T

\section{Introduction}

Dimensional metrology is the science of measuring geometric features of an object. Dimensional measurements are essential for the manufacturing industry to control the quality and efficiency of manufactured products by measuring the size, distance, angle, form, position or texture of components. With new manufacturing technologies in the field of precision engineering demands on the functionality of workpieces and their topography increased and thus also on metrology techniques. In order to understand today's dimensional metrology a brief historical review on its development, its importance for today's manufacturing industry and an outlook on the future conceptual developments of dimensional metrology are given.

\section{Evolution of Dimensional Metrology}

The origin of dimensional metrology is ascribed to the Egyptian culture. The Egyptians realized that they need a standardized measure for length to improve the accuracy of their buildings such as the pyramids, where several hundred people are working at the same time. The unit of length, they introduced, was related to the parts of the body of the reigning pharaoh and was called Royal Egyptian Cubit. The reference was the distance from Pharaoh's elbow to his fingertips plus the width of the palm of his hand [1]. The master royal cubit was made of granite and its copies which were given to the workers were either from wood or granite (Fig. 1).

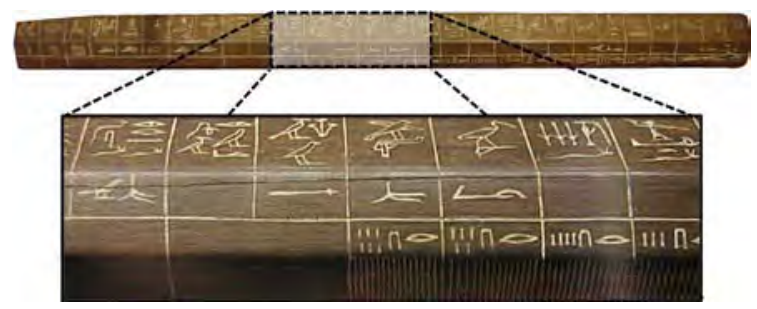

Fig. 1. Image of a Royal Egyptian Cubit (source: Jon Bodsworth, license: public domain).

Similar to the Royal Egyptian Cubit, each ruler has created his own units for measurement, which were not necessarily easily convertibly. As measuring systems mostly based on parts of human body, some units like "foot" or "inch" have retained their names to this day.

In addition to architecture also with surveying land new measurement techniques were developed. Reasons for surveying land were in par- 
ticular tax purposes, because levies were demanded in proportion to the area people owned or farmed. Then as now, the measurement procedure is still based on triangulation, i.e. a network of triangles is defined out whose area could be calculated. In the beginning the distances of the triangles were measured using rods or chains, later using measuring tapes and theodolites, and today using electronic range finders and satellite systems [2].

However, most important catalyst for dimensional metrology was the industrial production of manufactured goods, which is still today the driver of metrology. In the early days of manufacturing craftsmen ensured the proper function of individually manufactured workpieces by adjusting them to each other. This was a straight forward method, but also very laborintensive and therefore only feasible for limited number of parts. At the end of the $18^{\text {th }}$ Century, driven by rationalization efforts in production of muskets, Honoré Le Blanc in France and Eli Whitney in the U.S. popularized the idea of interchangeable parts. Instead of adjusting individual parts, similar parts, which were produced in cost-effective quantities, could be replaced arbitrary by "bin-picking" [3]. Since deviations from the nominal shape are inevitable due to the imperfections of the manufacturing process, also tolerances were defined and documented in the engineering drawing to limit the maximum deviations, in which the function interchangeability is fulfilled. Interchangeability or mating ability were the most important functional requirements in this time and are still today for workpieces in macro range, since the trend is manufacturing parts of a product at different locations. These developments in industry have forced metrology to develop new techniques and methods which allow checking the mating ability of workpieces in a short time without trial and error. In the $18^{\text {th }}$ and $19^{\text {th }}$ Century measurement devices like micrometer gauges or calipers were used to gain one-dimensionally measurements, i.e. only distances between two points were evaluated individually without regard of other measures. But with increasing demands on accuracy it was recognized that two-point measures might be insufficient to evaluate the function mating ability, e.g. when measuring the diameter of a shaft with nonnegligible form deviation [4].

A function-oriented approach for evaluating mating ability of a workpiece in a short time is the principle of limit gauging. Limit gauges (also called go/no-go gauge) were subject of a patent by William Taylor in 1905 and has since become known by the term Taylor's principle. It describes that the go gauge should be formed in this way that it assesses the tested form in its totality (maximum material condition, MMC) and the no-go gauge should only test individual dimensional features of the geometrical form of the workpiece (least material condition, LMC). Even today, these limit gauges are widely used in the production-related quality assurance.

With the invention of more precise measuring instruments in the first half of the $20^{\text {th }}$ Century, e.g. the length measuring machine of Carl Mahr, the measuring microscopes by Zeiss and Abbe, or the Werth profile projector, also twodimensional measurements were possible (Fig. 2). As well with form testing instruments and stylus instruments quantitative information about the degree of imperfectness of geometric features on workpieces could be determined.

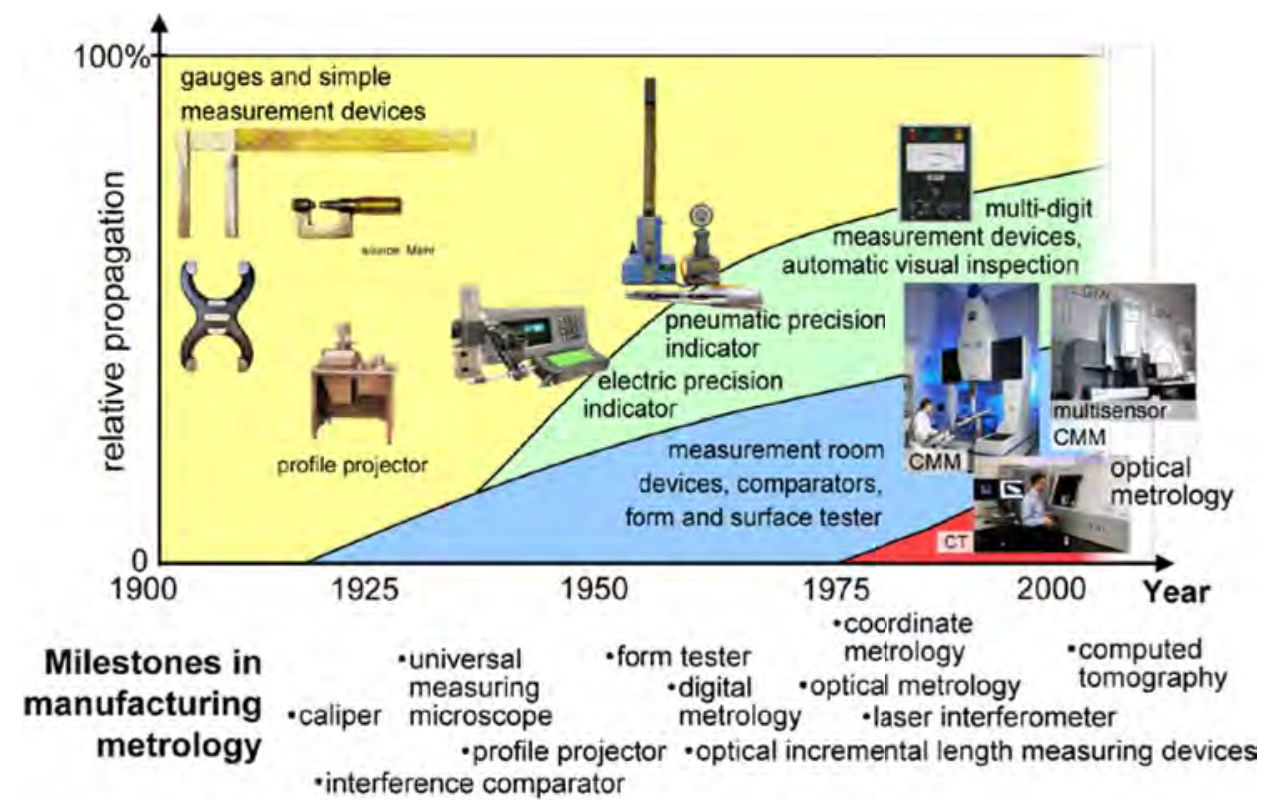

Fig. 2. Propagation of measurement devices in the $20^{\text {th }}$ century [5] 
Nevertheless, with ever more complex workpieces, where datums of the measured features are getting increasingly important, threedimensional measurements and their evaluation based on form and position tolerances were required. Metrologists were thinking more and more in coordinates and with first affordable computers the coordinate metrology was useable. Coordinate measuring machines (CMM), which first coming up in the 1970s, have the advantage that measures were taken no longer independently, but points on the surface of the workpiece were measured in one coordinate system and are so related to each other, so even complex measurement tasks can be managed, like the measurement of engine blocks. To test the conformance of a workpiece with the defined tolerances, geometric features (e.g. straight lines, planes, circles, spheres, cylinders etc.), which may even be linked (e.g. for calculation of distances between features) are calculated based on the probing points.

\section{Dimensional Metrology Today}

Our present dimensional metrology can be divided in three sub-disciplines: linear metrology (1D length measurement), angular metrology (measurement of angles) and geometrical metrology (3D coordinate measurement). With regard to the measurement data evaluation, there are two different approaches, called "hard dimensional metrology", which is based on hand tools and limit gauges, and "soft dimensional metrology", which is based on computerized evaluation of coordinates measured by tactile, optical, $\mathrm{x}$-ray tomographic or other measurement procedures [6]. While linear, angular and hard dimensional metrology has made no conceptual developments in recent decades, however, geometrical and soft dimensional metrology are rapidly growing areas.

Tactile coordinate metrology is still today the most versatile measurement principle and due to its standardization the reference for all other measurement principles. Today scale resolution of $1 \mathrm{~nm}$ and measurement uncertainty of $0.1 \mu \mathrm{m}$ is possible (Fig. 3). However other measurement principles offer different possibilities. Optical technologies enable measurements of the entire surface of a measurement object with an enormous point density, not possible with tactile CMMs. Additionally fragile workpiece can only be measured without destroying the object using optical sensors. In the following, some important examples of measurements techniques developed in recent decades are given. Moreover, further measurement techniques representing the state of the art of dimensional metrology are described in [4] and [7].

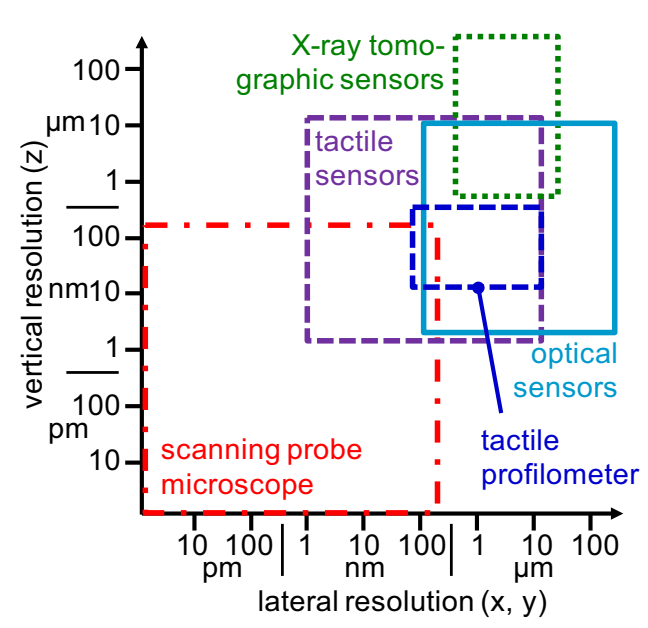

Fig. 3. Typical resolution of today's dimensional measuring sensors (based on [7])

Fringe projection is one of the techniques which offer fast, holistic and contact free sampling of complex surfaces with high point densities in relatively short time. They are especially used to measure complex freeform surfaces for inline process control and reverse engineering applications in automotive industry and aerodynamic investigations. Fringe projection systems are special triangulation systems working with coded illumination. Sequences of different patterns are projected onto the surface and observed under a defined angle. From the intensity modulation for each pixel of the camera, the height of the corresponding surface point can be calculated. During the measurement, at least one camera records a projected, parallel, sinusoidal fringe pattern under the calibrated triangulation angle $\mathrm{y}$, which is shown in Fig. 4. According to the triangulation principle, the distance $\Delta z$ is recorded as displacement $\Delta x$ on the camera(s).

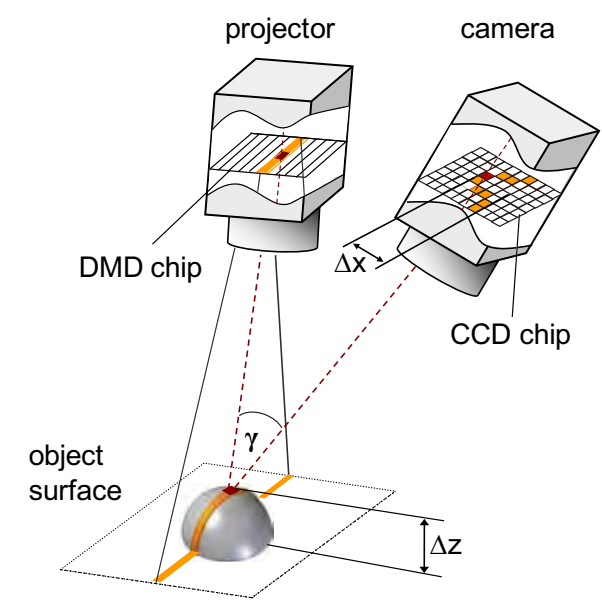

Fig. 4. Principle of fringe projection measurement

Quality control in the micro and nanometer range of optical devices (e.g. micro lens arrays), investigations of electronic circuits or inspection of silicon wafers are some of the 
applications in which the white light interferometer is used. It offers the possibility of $2 \mathrm{D}$ data acquisition with nanometer resolution. A brief description of the working principle is shown in Fig. 5. A beam splitter separates the light coming from the white light source into two beams. One of the beams reaches the object and the other the reference mirror. The objective is driven by a linear actuator element along the optical axis. During the movement in vertical direction (Z-direction), the intensity of the reflected light is stored for each pixel in the CCD element. The maximum of intensity modulation in the interference correlogram occurs at a position where the distance to the measuring object is equal to the distance to the reference surface. Finally, the height data together with the corresponding lateral coordinates give the topography of the surface [3].

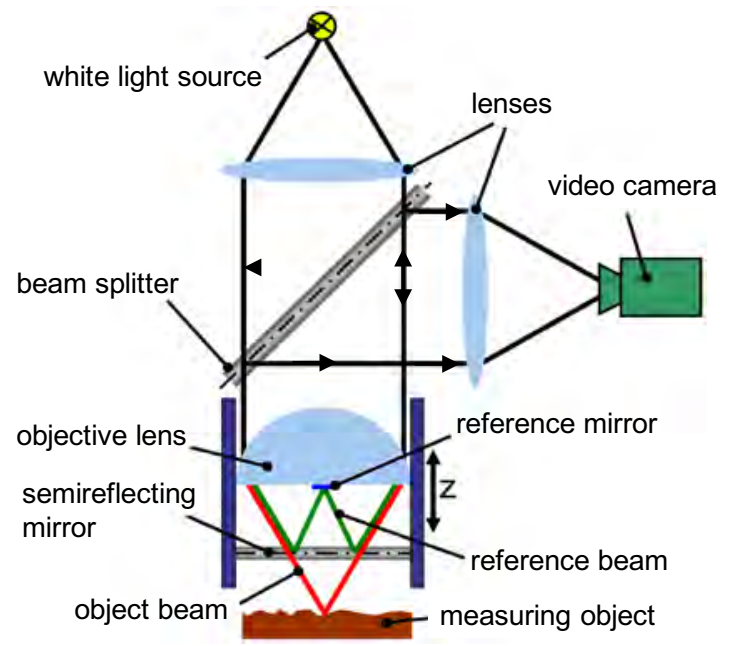

Fig. 5. Principle of white light interferometry

Due to the variety of workpieces and the very different demands on measurement accuracy, it is not possible to use a sensor generally preferred. For each individual application it has to be decided, which sensor fulfills the requirements at the best in total. Especially advantageous is the use of multi-sensor coordinate measuring systems, which combine different sensor principles in one device. They are calibrated to each other so a measurement in a common coordinate system is possible and an appropriate sensor can be selected for each micro or macro feature. Fig. 6 demonstrates how different features of a workpiece can be extracted with different sensors. With data fusion, i.e. the combination of information from different sensors, the total information of the measured workpiece increase. The scientific basis and some applications are shown in [7]. With multi-sensor data fusion it is possible to improve the accuracy of areal or volumetric measuring sensor, such as optical sensor or $\mathrm{x}$ ray computer tomography, by additional more accurate tactile measuring points, and to increase the whole measurement range.

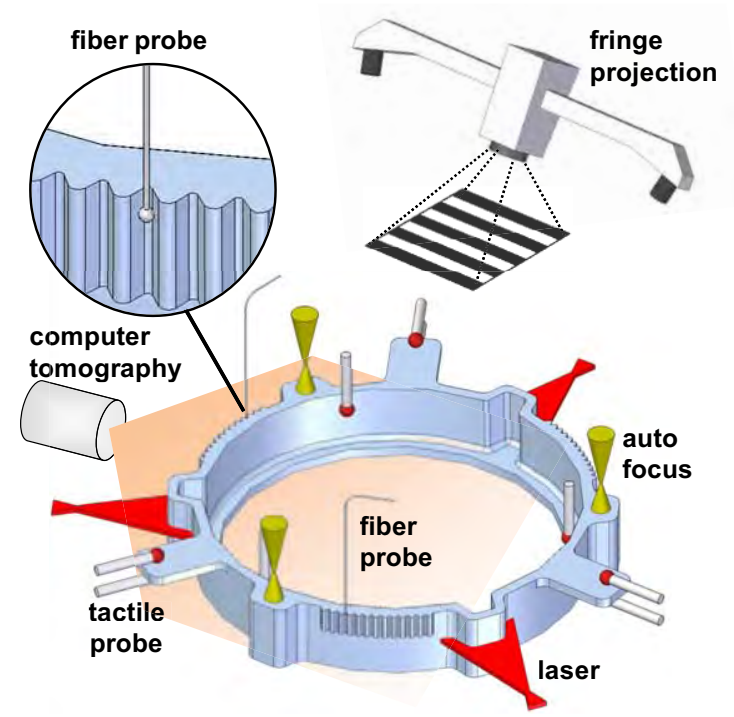

Fig. 6. Cooperative sensor integration in multisensorical CMMs

\section{Next Generation of Dimensional Metrology}

Nevertheless, despite of high-resolution and holistic geometric measurement data, in many cases it is not possible to make a clear statement about the functional ability of a workpiece. This problem is particularly exists at surface structures in the micro- and nanometer range because conventional concepts of dimensioning and tolerancing are solely geometry-oriented and standardized parameters are not sufficient to consider interaction with non-geometric parameters like adhesion and cohesion forces or hydrodynamic effects, which are dominant for functions like wettability or sliding. Here it is necessary to relate geometric variables with non-geometric variables, which makes the geometrical specification and verification with respect to a function-oriented conformity decision more complex and difficult.

As illustrated in Fig. 7, there are many intermediate steps to a conformity decision, which are all associated with uncertainties. Correlation uncertainties are present, if with the functional specification not all relevant requirements have been identified. Moreover, some information can get lost by the translation or simplification of the functional specification into a geometric specification according to the rules of ISO GPS (or ASME Y14.5). Thus, due to correlation uncertainties, in complex cases it is no longer sufficient only checking the conformity of a workpiece with the geometrical specifications to evaluate the functional ability of the workpiece. So for a function-oriented verification process a comparison of the measured features with the functional specifications should be sought. Es- 
pecially for functions that are influenced by micro-and nanostructures strongly (e.g. sliding, wettability, optical reflection etc.), focusing on functional specifications instead of simplified tolerances have advantages.

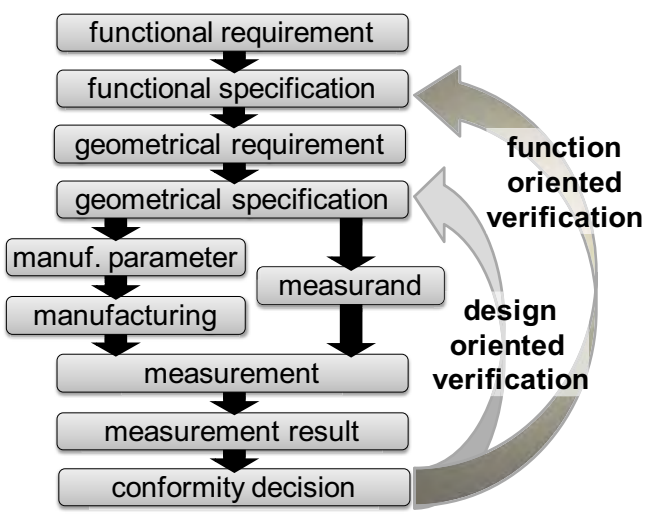

Fig. 7. Product development process, from product specification to verification

As described in [9], a general approach to solve this problem based on a parameterized mathematical-physical model of the function, which represents the causal interrelation between surface geometry and surface functionality to derive the required physical and geometrical properties for its design specification by simulations. So in future, the computer-aided analysis of measurement data will not be limited to standardized parameters, but also functional simulations (e.g. based on finite elements, fluid mechanics or tribological models) will be increasingly used in the verification process to predict the functional behavior based on the measured point cloud of the workpiece. Also the iMERA Metrology roadmap 'Dimensional metrology for micro-nano technology' [8] developed by European metrological institutes comes to the conclusion that one requirement of the future is the "modeling of functional propertied dependent on material and dimension".

In the following this approach is demonstrated by two examples of functional surfaces. One example is the microstructure of rolls in flexographic printing machines, which influenced the ink transfer behavior. The basic principles of ink transfer from a flexographic printing machine are based on the fact that initially the microstructure of an anilox roll is filled with ink in a chamber blade system and then a portion of the absorbed ink is transferred to a rubber roll. The rubber roll, on which the printing form is applied, then brings the ink to a printing substrate (paper, foil, etc.). The function of the anilox roll is to uniformly dye the printing form. However, this function cannot always be guaranteed. If the wet coating volume, which is transferred from the anilox roll to the printing form, is not constant, inhomogeneities may become appar- ent in the printed image. One reason for such inhomogeneities is the form deviation of the microstructure of the anilox roll which can cause different ink transfer behaviors. To monitor the production process of anilox rolls, function-oriented geometric features with tolerances are required to ensure the functional ability of the anilox roll structure, i.e. a highly homogeneous coating volume. As described in [10], with a model-based approach, which includes ink splitting models, wetting behavior and capillary effect are the most important factors that have a significant impact on the ink transfer function and should be determined. Also, geometrical parameters such as ground depth, bridge width, tri-helical engraving width and flank angle are derived. By setting up a model equation, the ink transfer behavior of different microstructures can be simulated. The simulation shows that the transferred ink volume and respectively the ink density is very sensitive at low $(<25 \mu \mathrm{m})$ deviations in depth, while variations in width have a 4-5 times smaller influence. At greater depth $(>30 \mu \mathrm{m})$ the impact of differences in depth has less influence on the ink transfer function, because more ink will not be transferred due to the capillary effect. So structures with low depth have to be toleranced closer to keep a homogeneous color application.

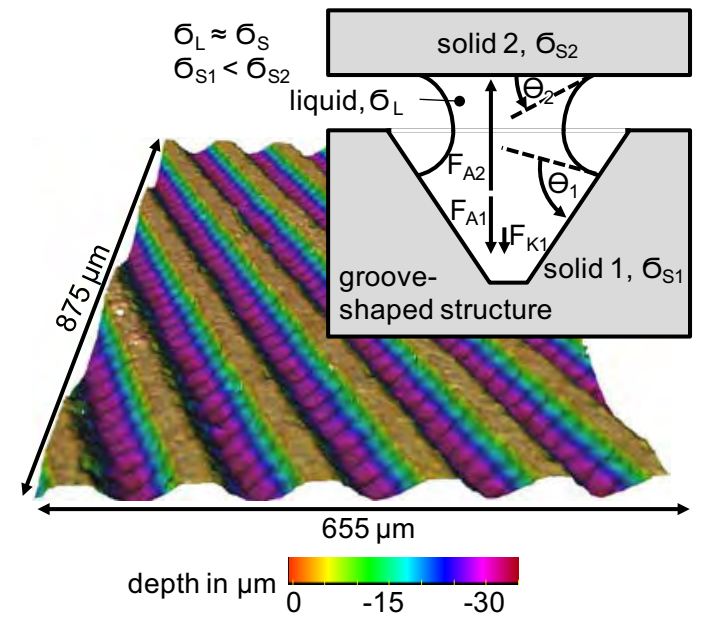

Fig. 8. Microstructure of an anilox roll and the used model for simulating the transferred ink volume (with wetting angle $\Theta$, adhesion force $F_{A}$, capillary force $F_{K}$, surface tension of the liquid $\sigma_{L}$, the solid $\sigma_{S}$ and the interfacial energy between liquid and solid $\sigma_{s}$ )

Another example of a surface function is metallic sealing of injectors. Although, in this application a surface structure is not specifically manufactured, a function-oriented measurement and evaluation is required to detect adverse functional features. Caused by manufacturing, rills are in the seating area, where the sealing ball or needle pressed against the nozzle body (Fig. 9). In particular, radial rills cause leakage, while concentric rills are of less importance. If radial 
microchannels are identified, one can then using a fluid-mechanical calculation according to Hagen-Poiseuille [11] to assess whether the measured diameter of a micro-channel, or the sum of all micro-channels are already critical for the function (e.g. simplified analytical calculation: pressure $p=300$ bar, max. leakage $5.0 \mathrm{ml} / \mathrm{min} \rightarrow$ max. diameter $3.1 \mu \mathrm{m})$.

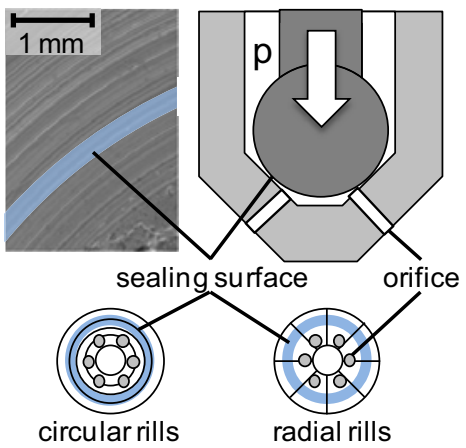

Fig. 9. Sealing of an injector by avoiding radial rills

By modeling and simulating the function of a component it is possible to evaluate its functional ability based on dimensional measurement data. This step leads to a better process understanding, a better control of function and an unambiguous product specification, based directly on function-oriented parameters instead of indirectly related dimensional parameters. Further examples of this function-oriented method are described in [9].

\section{Conclusion and Outlook}

Historically, dimensional metrology techniques have been developed based on the technological needs of the time. Dimensional metrology has been a key element in industrial progress and is still the substantial prerequisite of every engineering development. Measurement instruments and evaluation methods have evolved through many centuries, so with today's sophisticated measurement techniques extremely high resolved dimensional and topography measurements are possible. However, the technological needs for functionality of a workpiece changed, so that further functions like wettability, sliding or sealing getting important in addition to the usual function mating ability, which is well described by conventional tolerance concepts for the specification and verification of geometrical features. So these rules for dimensioning and tolerancing reach their limits especially for structures in the microand nanometer range, because the influence of non-geometrical parameters dominates more and more the functional ability. Since the functional ability is always influenced not only by one parameter, but by several, a mathematicalphysical model, which describes the technical function of the surface, is base for addressing the solutions of these identified open problems. Using the model like a kind of "virtual functional gauge" insufficient detailed standardized definitions and measurement evaluations can be omitted, whilst the functional ability is getting quantitatively predictable. Thus, model-based evaluation methods, which are able to simulate the functional behavior of a workpiece based on the measured data, will gain importance especially for micro- and nano-structures in the future.

\section{Acknowledgements}

This article is based on works within the project FunkProMikro, which was funded by the German Federal Ministry of Education and Research (BMBF), and the Transregional Collaborative Research Centre (Transregio) 73, which is funded by the German Research Foundation (DFG). The authors thank for support.

\section{References}

[1] J. Bucher, The metrology handbook, 1st edition, ASQ, 5-6 (2004).

[2] D. Flack, J. Hannaford, Measurement Good Practice Guide No. 80 - Fundamental Good Practice in Dimensional Metrology, NPL, 8-9 (2005).

[3] A. Weckenmann, Ö, Tan, The role of metrology in the exchange of goods and in the development of manufacturing technologies, Proceedings of $10^{\text {th }}$ ISMQC, 1-4 (05.-09.09.2010, Osaka, Japan).

[4] A. Weckenmann, J. Hoffmann, P. Krämer, Manufacturing Metrology - State of the Art and Prospects. Proceedings of $9^{\text {th }}$ ISMQC, 1-8 (21.24.11.2007, Chennai, India).

[5] A. Weckenmann, P. Philipp, G. Akkasoglu, Metrology - Base for Scientific Cognition and Technical Production, Advanced Materials Research, 498, 169-176 (2012)

[6] H. S. Nielsen, Taking Dimensional Metrology to the Next Level, Proceedings of the International Dimensional Metrology Workshop, (2005).

[7] A. Weckenmann, X. Jiang, K.-D. Sommer, U. Neuschaefer-Rube, J. Seewig, L. Shaw, T. Estler, Multisensor Data Fusion in Dimensional Metrology, Annals of the CIRP 58/1, 701-721 (2009).

[8] iMERA Metrology roadmaps (2011) www.technology-roadmaps.eu/doku.php?id=Micro_and_nano

[9] T. Wiedenhöfer, et al., Merkmalbasiert geregelte Fertigungsprozesse für die beherrschte Herstellung funktionaler Produkteigenschaften von mikrostrukturierten Bauteilen, Apprimus, (2012).

[10] A. Weckenmann, W. Hartmann, Funktionsorientierte Bewertung mikrostrukturierter Oberflächen, VDI-Berichte 2133, 207-216 (2011).

[11] J. Pfitzner, Poiseuille and his law. Anaesthesia 31 (2), 273-275 (1976). 